\title{
Papers
}

\section{Survival differences after stroke in a multiethnic population: follow-up study with the south London stroke register}

\author{
Charles D A Wolfe, Nigel C Smeeton, Catherine Coshall, Kate Tilling, Anthony G Rudd
}

\begin{abstract}
Objectives To identify ethnic differences in survival after stroke and examine the factors that influence survival.

Design Population based stroke register with follow-up.

Settings South London stroke register.

Participants 2321 patients with first stroke registered between January 1995 and December 2002.

Main outcome measures Sociodemographic factors, risk factors for stroke and their management, severity of stroke, and acute service provision factors. Survival analysis with Kaplan-Meier curves, log rank test, and Cox's proportional hazard model with stratification.

Results In univariable analyses of survival, outcome was better for black people than white people (median 33.7 v 20.0 months). After stratification by socioeconomic status, type of stroke, and Glasgow coma score, and adjustment for other potential confounders, being black was generally associated with better survival, taking into account the interaction between ethnicity and age, and ethnicity and prior Barthel score. Of the risk factors for stroke considered, current smoking (hazard ratio $1.21,95 \%$ confidence interval 1.01 to $1.45, \mathrm{P}=0.044$ ), untreated atrial fibrillation (1.36, 1.08 to $1.72, \mathrm{P}=0.009)$, untreated diabetes $(1.53,1.05$ to $2.22, \mathrm{P}=0.027)$, and treated diabetes (1.61, 1.27 to $2.03, \mathrm{P}<0.001)$ were associated with reduced survival.

Conclusion In general, black patients in a south London population with first ever stroke are more likely to survive than white patients, the exceptions being in those aged $<65$ and those with a prior Barthel score $<15$. Some pre-stroke risk factors that have the potential to be modified, including the appropriate treatment of existing health problems, have a strong impact on survival.
\end{abstract}

\section{Introduction}

Stroke is a major public health issue, and in many countries governments have set targets to reduce mortality without understanding the major influences on these rates. ${ }^{1}$ Black people have higher incidence and mortality for stroke than white people in the United Kingdom and the United States, ${ }^{2-4}$ with inconsistent evidence of disadvantage for black people in long term survival. In south London the average age of black people with stroke is 10 years lower than in the whole population. ${ }^{5}$ In Britain, Caribbean immigrants have the highest mortality from stroke. ${ }^{6}$ Howard et al estimated that in black men in the US up to $46 \%$ of the excess risk of death from stroke in men can be explained by socioeconomic class. ${ }^{7}$
The south London stroke register (SLSR) was established to investigate ethnic differences in the natural course of stroke. ${ }^{7}$ Although the use of race or ethnicity as a factor in medical research has been criticised, ${ }^{8}$ we used a well phenotyped stroke cohort to investigate ethnic differences in survival after a first stroke and to identify sociodemographic factors, pre-stroke risk factors and their management, indicators of stroke severity, and acute treatment factors associated with survival after stroke.

\section{Methods}

The population based stroke register recorded first ever strokes between January 1995 and December 2002 in patients of all age groups. ${ }^{79}$ We used the WHO definition of stroke and recorded ethnicity from the patient's own definition of ethnic origin (1991 census question) and socioeconomic class from the registrar general's codes based on occupation. ${ }^{79}$ Socioeconomic categories were grouped into non-manual, manual, and economically inactive. ${ }^{7}$ Ethnic origin was categorised into black (blackCaribbean, black African, and black other) and white. We restricted analysis to these categories only as numbers in other ethnic groups were small.

Classification of the pathological subtype (cerebral infarction, primary intracerebral haemorrhage, and subarachnoid haemorrhage) was based on results from at least one of brain imaging, analysis of cerebrospinal fluid, or necropsy examination. Cases without pathological confirmation of stroke subtype were unclassified. We also used the Oxford community stroke project clinical classification of stroke; cerebral infarction being categorised as total anterior cerebral, partial anterial cerebral, posterior cerebral, and lacunar. ${ }^{10}$

Data collected on pre-stroke risk factors included current smoking status, high alcohol intake ( $\geq 14$ units a week for women, $\geq 21$ units a week for men), hypertension (blood pressure $>140 / 90 \mathrm{~mm} \mathrm{Hg}$ ), atrial fibrillation, diabetes, ischaemic heart disease, and transient ischaemic attack. ${ }^{11}$

Management of risk factors before stroke was analysed by prescription of antihypertensive drugs; atrial fibrillation was subdivided into no treatment, aspirin, other antiplatelets, or warfarin; a history of transient ischaemic attack or ischaemic heart disease by treatment with aspirin; and diabetes by treatment with oral hypoglycaemics or insulin. Case severity variables before stroke included urinary incontinence, living alone before stroke, and prior Barthel index (categorised as 0-14 or 15-20). Case severity variables in the acute phase included uri-

Kaplan-Meier curves for black and white patients by age are on bmj.com 
Table 1 Risk factors, case severity, and service provision variables. Figures are numbers (percentages) of people unless stated otherwise

\begin{tabular}{|c|c|c|c|c|}
\hline Variable & All & White & Black & $P$ value \\
\hline Male & $1043 / 2135$ (49) & $827 / 1721 \quad(48)$ & $216 / 414 \quad(52)$ & 0.132 \\
\hline Mean (SD) age (years) & $71.2(14)$ & $73.3(13)$ & $62.4(15)$ & $<0.001$ \\
\hline Living alone & $737 / 1645$ (45) & $633 / 1338$ (47) & $104 / 307 \quad(34)$ & $<0.001$ \\
\hline Prior Barthel <15 & $148 / 2065(7)$ & 129/1666 (8) & $19 / 399$ & 0.038 \\
\hline Current smoker & $602 / 1893$ (32) & $517 / 1526(34)$ & $85 / 367(23)$ & $<0.001$ \\
\hline High alcohol intake & $259 / 1716$ (15) & $235 / 1380(17)$ & $24 / 336(7)$ & $<0.001$ \\
\hline Hypertension* & $1250 / 1983(63)$ & $964 / 1594(60)$ & $286 / 389(74)$ & $<0.001$ \\
\hline Atrial fibrillation* & $369 / 1996(18)$ & $348 / 1606 \quad(22)$ & $21 / 390(5)$ & $<0.001$ \\
\hline Diabetes* $^{*}$ & $331 / 2002(17)$ & $212 / 1609$ (13) & $119 / 393(30)$ & $<0.001$ \\
\hline TIA* & $278 / 1980(14)$ & $234 / 1589$ (15) & $44 / 391$ (11) & 0.077 \\
\hline Continence & $1280 / 2029(63)$ & $1001 / 1640(61)$ & $279 / 389(72)$ & $<0.001$ \\
\hline Failed swallow test & $877 / 1958$ (45) & $743 / 1586(47)$ & $134 / 372(36)$ & $<0.001$ \\
\hline GCS $\leq 8$ & $377 / 2057$ (18) & $311 / 1662$ (19) & $66 / 395(17)$ & 0.355 \\
\hline \multicolumn{5}{|l|}{ Cerebral infarction: } \\
\hline All & 1519/2067 (73) & $1241 / 1665$ (75) & $278 / 402(69)$ & $<0.001$ \\
\hline TACl & $331(16)$ & $277(17)$ & $54(13)$ & $<0.001$ \\
\hline $\mathrm{PACl}$ & $449(22)$ & 378 (23) & $71(18)$ & \\
\hline $\mathrm{POCl}$ & $223(11)$ & $187(11)$ & $36(9)$ & \\
\hline LACI & $516(25)$ & $399(24)$ & $117(29)$ & \\
\hline $\mathrm{PICH}$ & 282/2067 (14) & $210(13)$ & $72 / 402$ (18) & \\
\hline $\mathrm{SAH}$ & $116 / 2067 \quad(6)$ & 78/1665 (5) & $38 / 402(9)$ & \\
\hline Unclassified & $150 / 2067(7)$ & 136/1665 (8) & $14 / 402(3)$ & \\
\hline No hospital admission & $325 / 2135$ (15) & $271 / 1721$ (16) & $54 / 414(13)$ & 0.169 \\
\hline Stroke unit admission & $608 / 2106$ (29) & $446 / 1700(26)$ & $162 / 406 \quad(39)$ & $<0.001$ \\
\hline
\end{tabular}

IHD=ischaemic heart disease; TIA=transient ischaemic attack; GCS=Glasgow coma score; SAH=subarachnoid haemorrhage; TACI=total anterior cerebral infarction; PACl=partial anterior cerebral infarction; $\mathrm{POCl}=$ posterior cerebral infarction; $\mathrm{LACl}=$ lacunar infarction; $\mathrm{PICH}=$ primary intracerebral haemorrhage.

*Diagnosis in general practice or hospital records before stroke.

nary incontinence, ability to swallow, Glasgow coma score (categorised as $0-8$ or 9-15), pathological and clinical stroke subtype, and admission to a stroke unit, a general medical ward, or remaining in the community.

\section{Statistical methods}

Survival time was from date of stroke to date of death, confirmed by the Office for National Statistics. Patients with no record of death were censored at the end of 2003. Univariable analyses examined ethnic differences in sociodemographic factors, risk factors before stroke and their management, case severity, and service provision variables with age being compared with an unpaired $t$ test. Survival after the initial stroke was examined with unadjusted Kaplan-Meier survival curves, ${ }^{12}$ with comparisons between black and white groups made with the log rank test.

We used Cox proportional hazards survival analysis to investigate ethnic associations, adjusting for potential confounding factors. Exploratory analyses of increasing complexity were used, starting with a univariable comparison of ethnic groups, then in turn adding all sociodemographic variables, all prior risk factors and their management, then finally all factors associated with case severity, stroke subtype, and acute management. The analyses involved stratification by socioeconomic class, clinical stroke subtype, and Glasgow coma score. To allow for long term trends, we took account of the year of the stroke in the modelling in case there were trends over time.

Possible interactions between ethnicity and explanatory variables for survival were investigated by conducting separate analyses for both groups. Variables for which the degree of contribution to the survival model differed in these separate analyses were considered as potentially interacting with the ethnicity variable.
We examined assumptions for proportional hazards modelling using Schoenfeld residuals, with time dependent covariates being considered if the proportionality assumption was not met. $^{13}$

\section{Results}

The south London stroke register registered 2321 patients. Of these, $1721(74.1 \%)$ were white, $414(17.8 \%)$ black, and 186 $(8.0 \%)$ other groups or missing ethnicity. Table 1 shows sociodemographic factors, risk factors and their management, case severity, and acute service provision variables.

Regarding modifiable behavioural risk factors, a lower proportion of black people had a high alcohol intake and fewer were current smokers. High blood pressure and diabetes were more commonly observed in black people, with the reverse for ischaemic heart disease, atrial fibrillation, and transient ischaemic attack. Of those people with previously diagnosed medical conditions, a record of treatment for high blood pressure was more common for black people (180/286 (62.9\%) v 551/964 (57.2\%) white people). The same was true for ischaemic heart disease $(24 / 50(48.0 \%) v 148 / 371(39.9 \%))$. Treatment for transient ischaemic attack, however, was more common in white people than in black people (98/234 (41.9\%) v 13/44 $(29.6 \%))$.

Black patients were more likely to be admitted to the stroke unit, and similar proportions in both groups were managed in the community during the acute phase.

\section{Survival analysis}

The 2321 patients represented 6081 person years of time after stroke; 4253 person years for the 1721 white people and 1358 


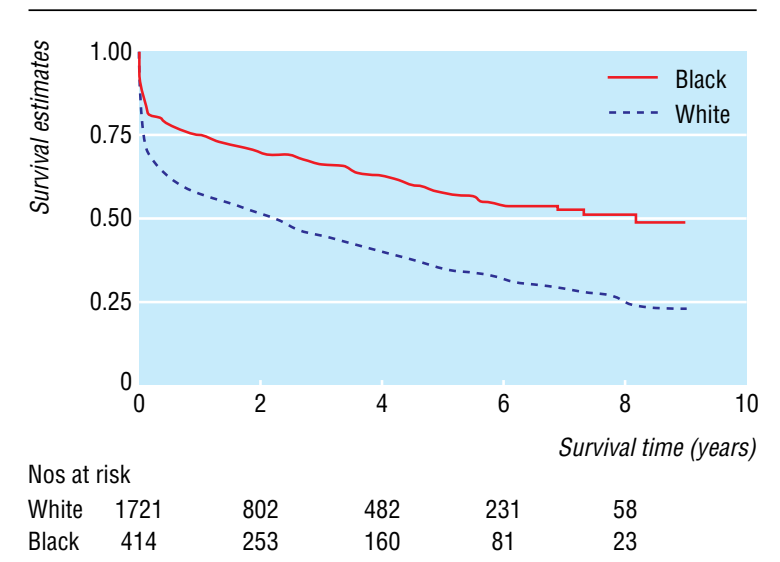

Kaplan-Meier survival estimates by ethnicity

for the 414 black people, with the remainder of other ethnic groups not analysed here. There were 1303 deaths (1097 and 166, respectively). Mean (median) survival was 31.2 (20.0) months for white people and 39.3 (33.7) months for black people. Case fatality was $275(16.0 \%)$ and $40(9.7 \%)$ at seven days and $567(32.9 \%)$ and $80(19.3 \%)$ at three months.

The Kaplan-Meier survival curve showed a clear difference between the two groups (figure), with white people having poorer survival (log rank test $\mathrm{P}<0.001)$. After adjustment for age and stratification for socioeconomic status and stroke subtype, this trend remained (hazard ratio $0.72,95 \%$ confidence interval 0.61 to $0.87, \mathrm{P}<0.001)$. In models without interaction terms, black ethnicity was a consistent indicator of better survival with a hazard ratio of about 0.7 at all stages of the proportional hazards modelling. At no stage did sex have an impact on survival. The survival curves for the two groups were almost identical in those aged $<65$, with a modest difference in favour of black people in those aged 65-74 and a substantial difference in those aged $\geq 75$ (see extra figures on bmj.com). Survival curves were similar for black and white people with a prior Barthel score $<15$, but there was a clear difference in favour of black people for those with a score of 15-20.

The effect of increasing age on survival was larger for white people than for black people. In addition, having a Barthel score $\geq 15$ was associated with a $83 \%$ reduction for the hazard ratio in black people but only $20 \%$ reduction in white people (see extra figures on bmj.com).

Table 2 shows the variables included in the final proportional hazards survival model (built up from the stepwise addition of variables and interaction terms). Ethnicity was highly significant (hazard ratio $24.80,95 \%$ confidence interval 4.70 to 130.87 ), but when we adjusted for the interaction between ethnicity and age (0.97, 0.95 to 0.99$)$ and prior Barthel score $(0.17,0.08$ to 0.36$)$, black people aged $\geq 65$ and with a prior Barthel score of at least 15 had a substantial survival advantage over white people.

Current smoking was an indication of poorer survival (1.21, 1.01 to 1.45 ). Patients with atrial fibrillation treated with warfarin alone fared worse $(1.83,1.09$ to 3.07$)$ as did similar patients who were untreated (1.36, 1.08 to 1.72$)$. Diabetes, treated (1.61, 1.27 to $2.03)$ or untreated $(1.53,1.05$ to 2.22$)$, was also an indicator of worse survival.

Initial continence was linked to better survival $(0.55,0.45$ to 0.67 ), and failing the swallow test was associated with worse survival $(1.49,1.23$ to 1.79$)$. Patients who were not admitted fared better $(0.59,0.45$ to 0.78$)$, and admission to a stroke unit had no impact on survival. As adjustment for the year of the stroke had no impact on the results we omitted this variable.
Table 2 Survival analysis stratified by Glasgow coma score ( $\leq 8$ or $9-15)$, subtype (cerebral infarction, PICH, SAH, unclassified), and socioeconomic status (non-manual, manual, economically inactive)

\begin{tabular}{|c|c|c|}
\hline Variable & Hazard ratio $(95 \% \mathrm{Cl})$ & $P$ value \\
\hline Black ethnicity & 24.80 (4.70 to 130.87$)$ & $<0.001$ \\
\hline Male & 1.13 (0.95 to 1.34) & 0.158 \\
\hline Age & 1.06 (1.05 to 1.07$)$ & $<0.001$ \\
\hline Living alone & 0.93 (0.80 to 1.09) & 0.327 \\
\hline Prior Barthel <15 & 1.20 (0.91 to 1.59) & 0.191 \\
\hline Current smoker & 1.21 (1.01 to 1.45) & 0.044 \\
\hline High alcohol intake & 0.88 (0.69 to 1.13$)$ & 0.327 \\
\hline \multicolumn{3}{|l|}{ Ischaemic heart disease: } \\
\hline Untreated & 1.19 (0.95 to 1.48$)$ & 0.122 \\
\hline Treated with aspirin & 0.95 (0.73 to 1.24) & 0.728 \\
\hline Untreated hypertension & 0.91 (0.75 to 1.11) & 0.357 \\
\hline Treated hypertension & 0.97 (0.81 to 1.17) & 0.772 \\
\hline \multicolumn{3}{|l|}{ Atrial fibrillation: } \\
\hline Untreated & $1.36(1.08$ to 1.72$)$ & 0.009 \\
\hline Aspirin only & 1.08 (0.87 to 1.45) & 0.590 \\
\hline Warfarin only & 1.83 (1.09 to 3.07$)$ & 0.023 \\
\hline Combined treatment & 1.08 (0.43 to 2.68) & 0.876 \\
\hline \multicolumn{3}{|l|}{ Diabetes: } \\
\hline Untreated & 1.53 (1.05 to 2.22) & 0.027 \\
\hline Treated & 1.61 (1.27 to 2.03) & $<0.001$ \\
\hline \multicolumn{3}{|l|}{ Transient ischaemic attack: } \\
\hline Untreated & 0.89 (0.67 to 1.16) & 0.381 \\
\hline Treated with aspirin & 1.25 (0.93 to 1.67) & 0.143 \\
\hline Continence & 0.55 (0.45 to 0.67$)$ & $<0.001$ \\
\hline Failed swallow test & 1.49 (1.23 to 1.79) & $<0.001$ \\
\hline No hospital admission & 0.59 (0.45 to 0.78$)$ & $<0.001$ \\
\hline Stroke unit admission & 0.99 (0.82 to 1.19) & 0.884 \\
\hline Ethnicity by age & 0.97 (0.96 to 0.99) & 0.008 \\
\hline Ethnicity by prior Barthel <15 & 0.17 (0.08 to 0.36$)$ & $<0.001$ \\
\hline
\end{tabular}

\section{Discussion}

In this study of ethnic differences in survival and the effect of sociodemographics, case severity, and clinical management factors on outcome in stroke we found that black patients are more likely to survive than white patients, even after we controlled for various factors. The south London stroke register (SLSR) is population based and hence unbiased with a well phenotyped cohort. This allowed us to control for potential confounding factors and identify clinical and social factors that can be used when planning services and tailoring individual clinical management plans.

The use of race and ethnicity in epidemiology and public health research has been debated hotly. Theoretical reasons for not using ethnicity per se as a variable have been well argued. ${ }^{14}{ }^{15}$ We used the UK census self definition of ethnicity, although the self reporting of ethnicity may change over time. ${ }^{14}$ The advantage is that the census definition is also used to categorise the local population estimates in this study. We amalgamated African Caribbean and African groups, yet biologically or culturally there may be no sense in such a grouping. To perform the analyses on African and African Caribbean groups separately will require a larger cohort. The census question could be supplemented with more detail on aspects of ethnicity-namely, cultural views of disease and health and access to health care. ${ }^{14}$ We have addressed some of these broader issues by determining socioeconomic status and quantifying uptake of effective stroke interventions. Bhopal suggests that by emphasising the negative aspects of health of minority groups, research may endanger their social standing. ${ }^{15}$ Despite an increased risk of disease, our analyses show a survival advantage in these black people. 


\section{Comparison with other studies}

Comparison of these findings with other reports with regard to ethnic or racial differences is problematic as no two studies use the same methods for determining the classification. The main reports on survival in "black" groups are in the US where studies of black Americans and Hispanics have been detailed but with more superficial adjustment for confounding factors. The findings are equivocal but suggest a survival disadvantage in black people. ${ }^{416}$ In the Northern Manhattan population based stroke study, unadjusted Kaplan-Meier analysis show a non-significant five year survival advantage in Caribbean Hispanics (67\%) and black Americans (61\%) compared with white people $(46 \%) .{ }^{17}$ Our study showed a clear advantage for black people, with five year survival being $57 \%$ for black people and $36 \%$ for white people.

The modifiable risk factors that increase the risk of stroke and recurrent stroke have been shown in population based stroke studies in the US and south London. ${ }^{11} 18$ Although comparison between differently classified ethnic groups in different cultures is problematic, striking similarities have been observed that have implications for primary and secondary prevention programmes. In the Manhattan studies hypertension, diabetes, and physical inactivity had high aetiological fractions in American black people and Caribbean Hispanics, which mirrors the prevalence of these risk factors in the south London population. Our analyses indicate improved use of appropriate medications for control of risk factors in black people after we controlled for age, although we did not assess dose and compliance. Improved management may reflect heightened awareness by black people and healthcare professionals to the risks of stroke. The ability of our analyses to assess the role of control of risk factors on survival may be hampered by a lack of power, although based on this study many of these factors had only a small impact.

\section{Possible confounding and bias}

One confounding factor may be the differences in case mix between groups. We adjusted for previous activity of daily living, Glasgow coma score, acute urinary incontinence, and swallowing deficits. Although this represents substantially more detail than most previous population based studies, there is the potential for residual confounding. Certainly in the acute period one might suspect that the observed survival differences may be explained by factors related to case mix, but adjustment for known confounders did not substantially change the hazard ratio for ethnicity, and it is unlikely that confounders that we did not adjust for here show the much stronger relations that would be needed to reverse the apparent observed trend in survival.

A possible cause of bias could have been if the register had missed either the more mild strokes in white people or the more severe strokes in black people (or both). In a previous study we did capture-recapture using the first two years of the register and found similar estimated completeness of cases in which patients survived to three months ( $90 \%$ for non-white and 88\% for white) but slightly higher estimated completeness for fatal cases in nonwhite patients $(90 \%)$ compared with fatal cases in white patients $(80 \%) .{ }^{19}$ If we apply those results to the current study, the true proportions of three month mortality would be 19\% for black people and 35\% for white people. Thus we can be reasonably sure that the black mortality advantage seen here is not due to ascertainment bias, although there is no accepted standard for case ascertainment for such population based registers and as such differential underascertainment in different ethnic groups may influence the interpretation of the survival data.

\section{What is already known on this topic}

The risk of stroke and mortality from stroke are higher in black ethnic groups compared with white groups

Analysis of survival after stroke between ethnic groups has rarely controlled for differences in socioeconomic status, management of pre-morbid risk factors, case mix, or acute management

\section{What this paper adds}

Black people with good mobility before a stroke and older black people have a substantial survival advantage over similar white people

The south London register has observed an increased risk of stroke but improved survival for most groups within the black population. After controlling for sociodemographic, case severity, and healthcare interventions we found a residual unexplained overall survival advantage in the black group that requires more detailed description of case mix and stroke subtype, including aetiological subtype. It seems that black people have better access to stroke unit care and more active management of some modifiable risk factors before stroke. A healthy migrant population from Africa and the Caribbean may confer some survival advantage, and more detailed measures of socioeconomic status and education need to be incorporated into future studies to identify the contribution of such factors on survival. Additional analysis of care after stroke-including location of care, rehabilitation, and ongoing health and social care, including secondary prevention and compliance with medication-will also shed light on these survival differences. McKevitt et al, using data from the south London stroke register, found that the provision of individual components of cure over one year after stroke varied for specific sociodemographic categories, but there was no consistent pattern of inequality with regard to ethnicity. ${ }^{20}$

We thank all the patients and their families and the healthcare professionals involved. Particular thanks go to all the fieldworkers who have collected data for the register since 1995 .

Contributors: CDAW participated in the design and refining of the study methods, analysed the data, wrote the paper, and is guarantor. AGR, KT, and NCS participated in the refining of the study methods, analysis of the data, and drafting of the paper. CC participated in the refining of the study methods and of the paper.

Funding: Northern and Yorkshire NHS research and development programme in cardiovascular disease and stroke, Charitable Foundation of Guy's and St Thomas' Hospitals, Stanley Thomas Johnson Foundation, Department of Health, UK.

Competing interests: None declared.

Ethical approval: Ethical approval was from the ethics committees of Guy's and St Thomas' Hospital Trust, King's College Hospital, Queens Square, and Westminster Hospital.

Secretary of State for Health. Our healthier nation: a strategy for health in England. London: Stationery Office, 1997 .

2 Howard G, Anderson R, Sorlie P, Andrews V, Backlund E, Burke GL. Ethnic differences in stroke mortality between non-Hispanic whites, Hispanic whites, and blacks. The national longitudinal mortality study. Stroke 1994;25:2120-5.

Gillum RF. Stroke mortality in blacks. Disturbing trends. Stroke 1999;30:1711-5.

4 Rosamond WD, Folsom AR, Chambless LE, Wang CH, McGovern PG, Howard G, et al. Stroke incidence and survival among middle-aged adults: 9-year follow-up of the atherosclerosis risk in communities (ARIC) cohort. Stroke 1999;30:736-43.

5 Wolfe CD, Rudd AG, Howard R, Coshall C, Stewart J, Lawrence E, et al. Incidence and case fatality rates of stroke subtypes in a multiethnic population: the south London stroke register. J Neurol Neurosurg Psychiatry 2002;72:211-6.

6 Balarajan R. Ethnicity and variations in the nation's health. Health Trends 1995;27:114-9. 
7 Howard G, Russell GB, Anderson R, Evans GW, Morgan T, Howard VJ, et al. Role of social class in excess black stroke mortality. Stroke 1995;26:1759-63.

8 Kaufman JS, Cooper RS. Commentary: considerations for use of racial/ethnic classification in etiologic research. Am J Epidemiol 2001;154:291-8.

9 Stewart JA, Dundas R, Howard RS, Rudd AG, Wolfe CD. Ethnic differences in incidence of stroke: prospective study with stroke register. BMJ 1999;318:967-71.

10 Bamford J, Sandercock P, Dennis M, Burn J, Warlow C. Classification and natural history of clinically identifiable subtypes of cerebral infarction. Lancet 1991;337:1521-6.

11 Hajat C, Tilling K, Stewart JA, Lemic-Stojcevic N, Wolfe CD. Ethnic differences in risk factors for ischemic stroke: a European case-control study. Stroke 2004;35:1562-7.

12 Campbell MJ. Statistics at square two. Understanding modern statistical applications in medicine. London: BMJ Books, 2001

13 Collett D. Modelling survival data in medical research. 2nd ed. Boca Raton: Chapman and Hall/CRC, 2003.

14 Comstock RD, Castillo EM, Lindsay SP. Four-year review of the use of race and ethnicity in epidemiologic and public health research. Am J Epidemiol 2004;159:611-9.

15 Bhopal R. Is research into ethnicity and health racist, unsound, or important science? BMJ 1997;314:1751-6.

16 Gillum RF, Mussolino ME. Education, poverty, and stroke incidence in whites and blacks: the NHANES I epidemiologic follow-up study.J Clin Epidemiol 2003;56:188-95.

17 Hartmann A, Rundek T, Mast H, Paik MC, Boden-Albala B, Mohr JP, et al. Mortality and causes of death after first ischemic stroke: the Northern Manhattan stroke study. Neurology 2001;57:2000-5
18 Sacco RL, Boden-Albala B, Abel G, Lin IF, Elkind M, Hauser WA, et al. Race-ethnic disparities in the impact of stroke risk factors: the northern Manhattan stroke study. Stroke 2001;32:1725-31.

19 Tilling K, Sterne JA, Wolfe CD. Estimation of the incidence of stroke using a capturerecapture model including covariates. Int J Epidemiol 2001;30:1351-9.

20 McKevitt C, Coshall C, Tilling K, Wolfe C. Are there inequalities in the provision of stroke care? Analysis of an inner-city stroke register. Stroke 2005;36:315-20.

(Accepted 27 May 2005)

doi $10.1136 /$ bmj.38510.458218.8F

Division of Health and Social Care, King's College London, London SE1 3QD

Charles D A Wolfe professor of public health medicine

Nigel C Smeeton lecturer in medical statistics

Catherine Coshall research associate

Department of Social Medicine, Clifton, Bristol BS8 2PR

Kate Tilling senior lecturer in medical statistics

Department of Ageing and Health, St Thomas' Hospital, London SE1 7EH

Anthony G Rudd consultant stroke physician

Correspondence to: C D A Wolfe charles.wolfe@kcl.ac.uk 